\title{
New Approach for Monitoring a Direct Laser Interference Patterning Process Using a Combination of an Infrared Camera and a Diffraction Measurement System
}

\author{
Nikolai Schröder ${ }^{* 1}$, Sascha Teutoburg-Weiss ${ }^{1}$, Germán Vergara ${ }^{2}$ and Andrés Fabián Lasagni ${ }^{1,3}$ \\ ${ }^{1}$ Technische Universität Dresden, Institut für Fertigungstechnik, George-Bähr-Str. 3c, \\ 01069 Dresden, Germany \\ ${ }^{2}$ New Infrared Technologies, Calle Vidrieros, 30 - Nave 2, Boadilla del Monte, \\ 28660 Madrid, Spain \\ ${ }^{3}$ Fraunhofer-Institut für Werkstoff- und Strahltechnik IWS, Winterbergstr. 28, \\ 01277 Dresden, Germany
}

*Corresponding author’s e-mail: nikolai.schroeder@tu-dresden.de

\begin{abstract}
Recently, process monitoring emerges as a breakthrough technology in industrial laser machines applications to enhance process stability and economic efficiency while ensuring high-quality processing parts and significantly reducing scrap rate. Furthermore, the latest advances in monitoring systems open a broad range of new opportunities to increase the capabilities of laser surface structuring. In this study, stainless steel and aluminum substrates are structured with a line-like geometry by Direct Laser Interference Patterning. A high-speed infrared camera is used to detect the thermal effects throughout the laser process. Simultaneously, a diffraction measurement system is implemented to analyze the quality of the fabricated periodic patterns by comparing the diffraction order characteristics. This specific combination of the systems allows a remarkably high-performance process monitoring and quality assurance. The obtained results reveal a correlation between the signals detected by the infrared camera and the intensity of the diffraction orders recorded with the quality of the surface reached.
\end{abstract}

DOI: $10.2961 /$ jlmn.2021.02.2009

Keywords: direct laser interference patterning, DLIP, pulsed laser, stainless steel, aluminum, monitoring, heat accumulation, infrared camera, diffraction, scatterometry

\section{Introduction}

Currently, functional surfaces resulting from structured materials with features in the micrometer and sub-micrometer range can be efficiently produced using industrial laser microprocessing systems equipped with ultra-short pulsed laser sources. These generated textured surfaces are often designed to mimic the unique structures that can be found in many natural examples such as plants, animals and microorganisms that exhibit outstanding properties. These attributes can be related to hierarchical microscale features and surface chemistry [1-3]. These engineering-specific functionalities include, for instance superhydrophobic properties [4-6], self-cleaning and antibacterial behavior [7-9] and light reflexion materials among others [10,11].

One of the most advanced structuring techniques capable to create well-defined periodic micro/nanopatterns on a variety of materials with high throughput is Direct Laser Interference Patterning (DLIP) [12-14]. In DLIP, a coherent and pulsed laser beam is split into two or more sub-beams that are subsequently superimposed on the surface of the workpiece. This results in an interference pattern in which the material surface is selectively ablate or modify at the interference maxima positions. Furthermore, while the geometry of the interference pattern can be changed by using a different number of sub-means as well as by controlling the laser beam polarization, the intercepting angle of the beams determine the periodic distance (spatial period) of the pattern. Moreover, the method has been shown to produce textures with feature sizes down to $170 \mathrm{~nm}[15,16]$. As in several manufacturing methods, both efficiency and process safety are of great economic importance [17]. Therefore, process monitoring is a highly valuable tool to guarantee the quality of the manufacturing process in industrial production.

Monitoring methods of laser processes and the manufactured parts are essential to improve the process stability by optimizing reproducibility as well as increasing the quality of the outcoming items [18]. Moreover, these methods can be used in laser processing to generate a comprehensive understanding of the underlying processes such as analyzing the temperature distribution field in additive manufacturing $[19,20]$, studying the optical emission of plasma plumes [21], and evaluating the weld penetration depth in laser welding [22].

As in other applications, where an ultra-short pulsed laser system is used, the heat accumulated by the process might substantially influence the conditions of the fabricated structures. Furthermore, the thermal effects exhibit a strong dependency with the material properties and the process parameters utilized [23]. Infrared cameras have shown the ability to monitor these effects during short and ultra-short laser 
processing and can be implemented for improving the stability of a process [24-26]. In addition, to classify the condition of a patterned area, the produced topography has to be characterized and analyzed. However, this is particularly challenging to realize in a real-time process, for instance by using typical topographical characterization methods such as confocal microscopy (CM) and scanning electron microscopy (SEM) $[27,28]$. Hence, despite their strong potential in high-resolution imaging, both methods are limited in terms of their applicability for larger areas.

A promising strategy to close the gap between topographical information and measurement time is the integration of a scatterometry-based system [29-31]. This system allows a high-speed evaluation of the topography of the patterned areas even in the course of the laser treatment, and thus permitting control of the stability and efficiency of the DLIP process. The concept of the method is based on measuring and analyzing the light diffracted from the lasertreated surfaces. When a coherent light source irradiates a surface with a periodic structure, the reflected light beams picture a diffraction pattern. If the incident irradiation is normal to the surface of the sample, the captured image includes different diffraction orders, which can be mathematically described using (1):

$$
\sin \theta_{m}=m \cdot \frac{\lambda}{\Lambda}
$$

where $\theta_{m}$ is the angle of diffraction for the $m$-diffraction order relative to the surface, $\lambda$ is the wavelength of the incident light beam and $\Lambda$ is the spatial period of the structured linelike pattern on the sample surface [32]. Thus, line-like textures with larger spatial periods produce shorter diffraction angles and vice versa.

A combination of a scatterometry system with infrared cameras can be used to further enhance the capabilities of the monitoring strategy in order to detect flaws during the laser treatment. However, although there is a range of monitoring technologies available on the market, to the best of our knowledge, they have rarely been used to optimize the DLIP process.

In the present study, stainless steel and aluminum substrates were structured by DLIP varying different process parameters such as laser fluence and repetition rate. An uncooled high-speed mid-wavelength infrared (MWIR) camera was used to monitor the DLIP experiments to detect and analyze possible heat accumulation effects. The MWIR camera was integrated with a diffraction measurement unit in order to increase the capability of the control systems to detect possible flaws associated with the quality of the periodic textures produced. Afterwards, for the sake of comparison of the two methods the topography of the laser treated samples were characterized with CM and SEM methods.

\section{Experimental procedure}

\subsection{Materials}

Stainless steel sheets (X5CrNi18-10, 1.4301) with a thickness of $0.8 \mathrm{~mm}$ and aluminum sheets (2024-T351) with $1.0 \mathrm{~mm}$ thickness were used in this study. All samples were electropolished in order to avoid effects of the initial surface roughness on the process evolution. The surface roughness $S_{a}$ for stainless steel and aluminum were $9.5 \mathrm{~nm} \pm 1.1 \mathrm{~nm}$ and $159.3 \mathrm{~nm} \pm 30.3 \mathrm{~nm}$, respectively. Before the laser experiments, all metallic samples were gently rinsed with ethanol in order to remove any contaminations.

\subsection{Laser structuring setup}

The structuring process of the metallic samples was performed in a DLIP workstation including an ultra-short pulsed laser source (PX200-2-GFH, EdgeWave GmbH, Germany) and an optical DLIP head (Fraunhofer IWS, Dresden, Germany) for two-beam interference patterning (see Figure 1). The $12 \mathrm{ps}$-pulsed laser system operated at $532 \mathrm{~nm}$ wavelength with a maximum repetition rate of $100 \mathrm{kHz}$. A positioning stage system (PR0165-300, Aerotech, USA) accomplished the movement of the sample under the DLIP head in $\mathrm{x}$ - and y-directions. This experimental setup allowed the production of line-like periodic patterns with a defined repetitive distance of $4.5 \mu \mathrm{m}$ by overlapping the laser beams at an angle $\alpha$ of $6.8^{\circ}$.

The pulse-to-pulse overlap $o_{P}$, the repetition rate $f_{R}$ and the laser fluence $F$ were varied in order to obtain different structuring results. The repetition rate was adjusted to the speed of the positioning stage system in order to obtain different pulse overlaps. For each used condition, areas of approximately $40 \times 5 \mathrm{~mm}^{2}$ were structured with the line-like pattern geometry by keeping the hatch overlap at $0 \%$. Then, the laser spot was shifted until 30 parallel lines were produced. Table 1 shows the different process parameters used for the structuring experiments.

Table 1 Applied process parameter for the DLIP surface structuring of the metal samples $\left(f_{R}\right.$ : repetition rate, $o_{P}$ : pulse-topulse overlap, $F$ : laser fluence)

\begin{tabular}{ccc}
\hline$f_{R}(\mathrm{kHz})$ & $o_{P}(\%)$ & $F\left(\mathrm{~J} / \mathrm{cm}^{2}\right)$ \\
\hline 100 & $99 \ldots 99.67$ & $0.21 \ldots 0.69$ \\
80 & $99 \ldots 99.67$ & $0.18 \ldots 0.45$ \\
\hline
\end{tabular}

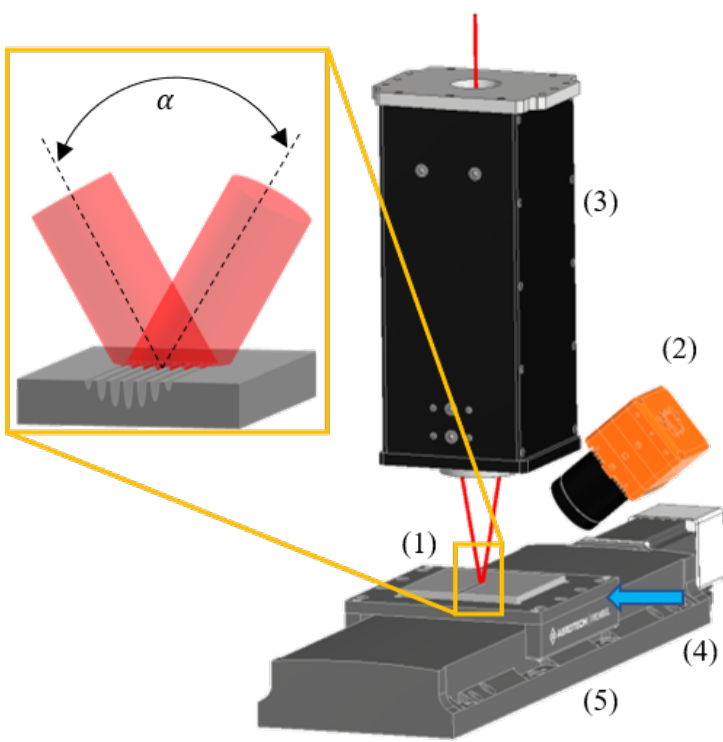

Fig. 1 Experimental setup for structuring and monitoring the heat accumulation during a two-beam-DLIP treatment of metallic samples: (1) interference volume produced by the overlap of two laser beams at an angle $\alpha$ producing a line-like structure; (2) midwave infrared camera; (3) DLIP head; (4) cross jet gas (compressed air); (5) positioning stage system. 


\subsection{Monitoring systems}

A midwave infrared camera (TACHYON 16k, New Infrared Technologies NIT, Spain) in an off-axis configuration was used to detect the thermal effects at the material surface occurring during the laser treatment. The camera was equipped with an objective lens with a focal length of $35 \mathrm{~mm}$ and a protecting silicon window for filtering the visible and near-infrared light including the reflection of the laser beam. The working distance between the sample and camera was $100 \mathrm{~mm}$ at an angle of $45^{\circ}$ to the vertical direction. The capturing process was set to a constant frame rate of $1 \mathrm{kHz}$ and a fixed integration time of $200 \mu \mathrm{s}$. Depending on the applied process parameters, every experiment showed specific characteristics of the infrared signal. Figure 1 shows the configuration employed for the infrared camera.

The laser-treated surfaces were analyzed (ex-situ) with a specifically designed diffraction measurement system (TU Dresden, Germany) already described elsewhere [30]. The Figure 2 shows the schematic setup of the diffraction measurement system including the required optical components, the camera as well as the coherent light source (e.g. a laser pointer). The method is based on irradiating the laser-treated area with a low-power laser source operating at a wavelength of $532 \mathrm{~nm}$. Afterwards, the reflected radiation passes an optical system with a charge-coupled device (CCD) camera that constantly captures the diffraction patterns, which are correlated to the topography of the produced structure [30]. The collected images can be rapidly analyzed by computer algorithms.

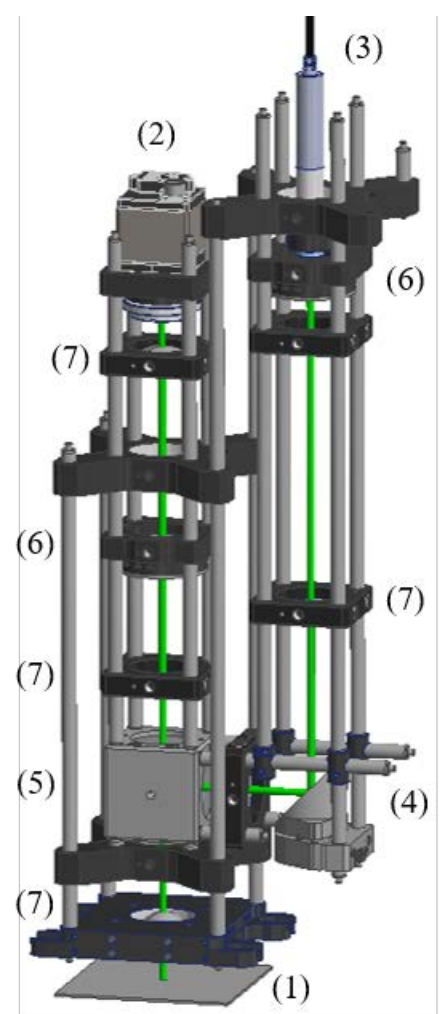

Fig. 2 Representation of the diffraction measurement system with laser structured sample at the bottom (1). The beam path of the illumination beam and the reflected diffraction pattern from the treated surface are also shown. The system includes the use of a (2) CCD camera, (3) a laser pointer, (4) a coupling mirror, (5) a beam splitter, (6) two polarizers and (7) a lens.
All captured images were analyzed using ImageJ (National Institute of Health, Bethesda, USA) software.

\subsection{Surface characterization}

The topography of the processed samples was characterized using CM with a 20x magnification objective (Sensofar $\mathrm{S}$ Neox, Spain). The optical and vertical resolutions of the used objective were $310 \mathrm{~nm}$ and $8 \mathrm{~nm}$, respectively. The software SensoMap ${ }^{\circledR} 7.3$ (Sensofar, Barcelona, Spain) was used to analyze the measured topography data. Likewise, high resolution pictures of the surfaces were obtained with a SEM (Zeiss Sigma 300, Germany) at an operating voltage of $6.0 \mathrm{kV}$ and work distance of $5.5 \mathrm{~mm}$.

\section{Results and discussion}

Confocal and SEM images of the obtained line-like pattern were analyzed in order to achieve a qualitative evaluation of the surface quality (see Figure 3). As it can be seen, the good surface condition was indicated by a well-defined line-like pattern. The surface topography of the sample showed a homogeneous structure without any remarkable solidified material. The line-like structures had a maximum depth of $1.99 \mu \mathrm{m} \pm 0.35 \mu \mathrm{m}$ for stainless steel and $2.12 \mu \mathrm{m} \pm 0.28 \mu \mathrm{m}$ for aluminum, respectively. In contrast, the bad quality surface exhibited an extensive amount of resolidified material on the line-like pattern and had a deteriorated structure. The amount of the remolten material can strongly influence the orientation and uniformity of the linelike structure.

For the quantitative description of the quality of a periodic structure, an analytical surface parameter was established in this work. In particular, due to the directionality of the produced lines, the texture (or pattern) orientation of the produced periodic structure can be utilized as has been already shown in other studies [33]. The isotropy of a surface is a spatial area roughness parameter that indicates a criterion for uniformity of surface patterns in the different directions. A three-dimensional surface characterization based on an autocorrelation analysis of the surface texture computes the isotropy. The value allows the distinction between the well-aligned from the misaligned surfaces. The texture aspect ratio $S_{t r}$ defines the isotropy $I$ that can be calculated with (2):

$$
I[\%]=100 \cdot S_{t r}
$$

This parameter can vary between 0 and $100 \%$. In one extreme case a perfectly isotropic surface has identical properties independent from the measurement direction and it shows no prominent textures on the surface. In this case, the texture aspect ratio value is 1 and, consequently, the isotropy is $100 \%$. On the other extreme case, if the isotropy value is $0 \%$, the surface presents a totally anisotropic structure with an specific orientation or periodicity [34], which is the case of perfect periodic line-like structure. The percentage of isotropy of all laser processed samples were determined and correlated to the CM images of the corresponding surface pattern.

Due to the strategy used to process the samples, each laser structured line (containing by the interference pattern) could have an isotropy value that can differ from the other 
lines depending on the accumulated heat. Therefore, to establish a statistical analysis, the isotropies of the $1^{\text {st }}, 15^{\text {th }}$ and $30^{\text {th }}$ line were calculated from the CM images and their average value was used as a descriptive parameter of the homogeneity and quality of the whole laser treated area. Based on the evaluation of the CM images of the treated examples (see Figure 3a) a threshold isotropy I value was defined, corresponding to the condition in which any melt or deterioration of the line-like pattern was observed. For both used materials, stainless steel and aluminum, the determined threshold isotropy value was $2.3 \%$ which was significantly close to the theoretical value for a perfect line-like structure $(0 \%)$.

In addition to the SEM and CM of selected structures, Figure 3 shows for the different surface conditions the calculated isotropy values. As it can be seen, for lower fluences and overlaps, both materials presented a homogenous pattern and the calculated isotropies $I$ were $1.94 \%$ and $1.97 \%$ for stainless steel and aluminum, respectively. By using higher fluences and overlaps, the laser-treated surfaces exhibit a considerable excess of resolidified material over the structure. In this case, the amount of resolidified material increases with the number of structured lines $\left(1^{\text {st }}\right.$ line to $30^{\text {th }}$ line) due to the accumulated heat in the material that occurred during the laser treatment [35]. Therefore, the isotropy for a bad surface quality raises with the number of lines. That implies a huge deviation of the mean isotropy value.

The condition of the surfaces also reflects in the corresponding infrared intensity captured by the MWIR camera. Figure 4 shows exemplarily the maximum intensities of the detected infrared signal by the MWIR camera for stainless steel and aluminum as a function of the line length for two different processing conditions. At the start of the laser process, the infrared signal for the stainless steel sample treated with a laser fluence of $0.59 \mathrm{~J} / \mathrm{cm}^{2}$ increases rapidly (Figure 4a). This indicates that the energy introduced in the sample by the laser irradiation did not dissipate fast enough. This could be explained by the high energy levels used as well as the relative low thermal conductivity of the stainless steel material. Consequently, heat started to accumulate. After the substrate was translated approximately $20 \mathrm{~mm}$, the infrared signal dropped to a constant level. The behavior of the infrared signal at this point could be based on the fact that the

\section{Good surface quality}

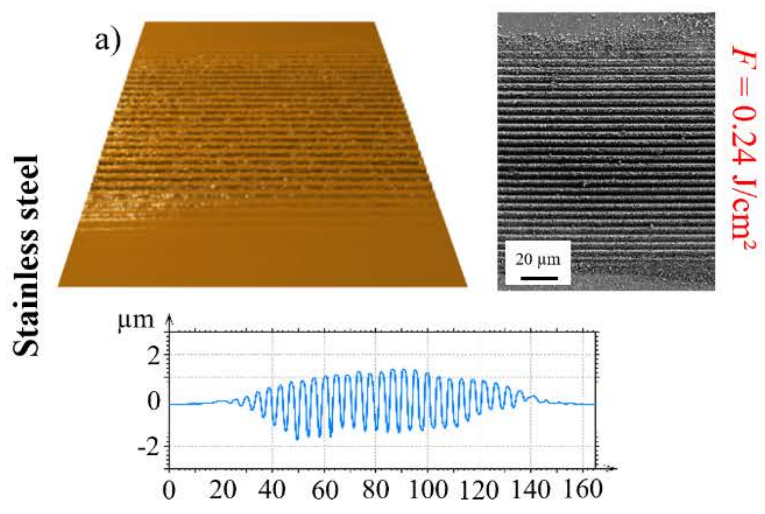

Bad surface quality

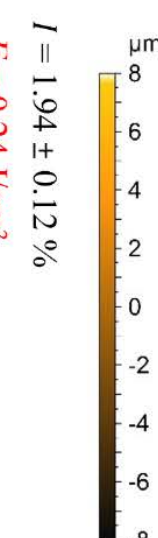

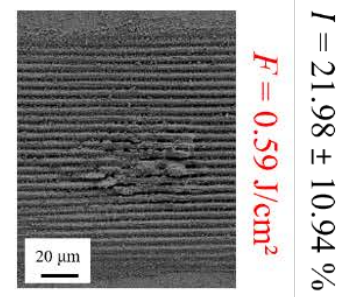
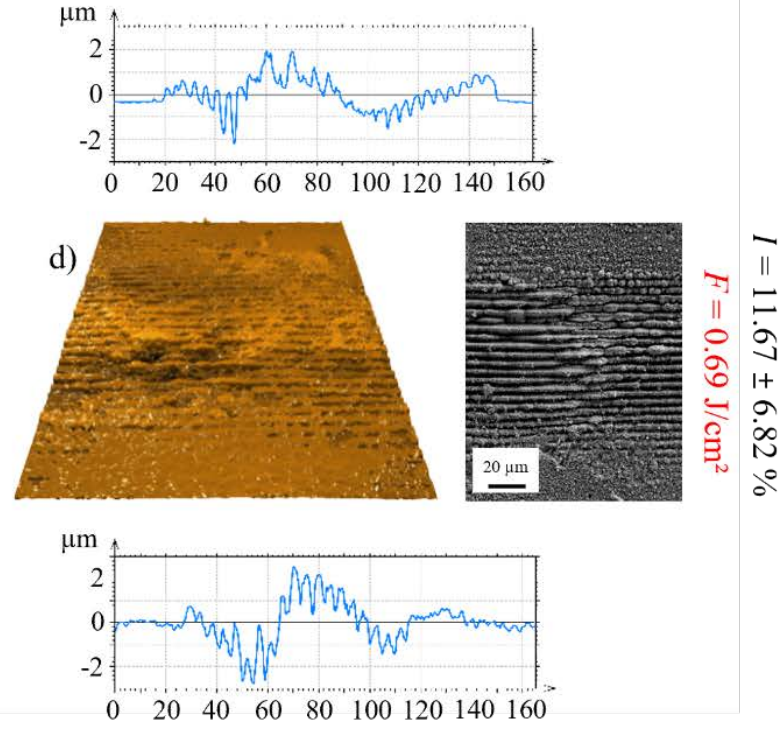

Fig. 3 Confocal microscope and scanning electron microscope images of the produced structure on stainless steel (a, b) and aluminum samples (c, d) processed at: (a) $F=0.24 \mathrm{~J} / \mathrm{cm}^{2}$ and $o_{P}=99.67 \%$ showing a good surface quality with a homogeneous pattern and a mean isotropy of $1.94 \%$, (b) $F=0.59 \mathrm{~J} / \mathrm{cm}^{2}$ and $o_{P}=99.67 \%$ showing a bad surface quality with an significant amount of resolidified material on the line-like pattern and a mean isotropy of $21.98 \%$, (c) $F=0.28 \mathrm{~J} / \mathrm{cm}^{2}$ and $o_{P}=99.67 \%$ showing a good surface quality with a homogeneous pattern and a mean isotropy of $1.97 \%$, and (d) $F=0.69 \mathrm{~J} / \mathrm{cm}^{2}$ and $o_{P}=99.67 \%$ showing a bad surface quality with an extensive amount of resolidified material on the line-like pattern and a mean isotropy of $11.67 \%$. The obtained mean roughness profiles of the structures are shown below the corresponding microscopy images. The SEM images do not reflect the same surface position that is shown in the CM images. 
a)

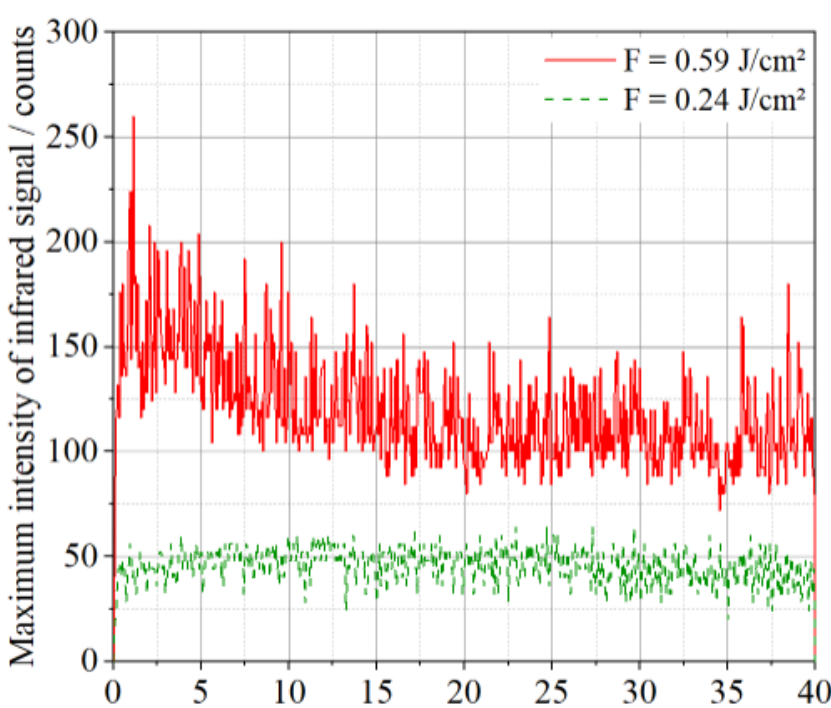

b)

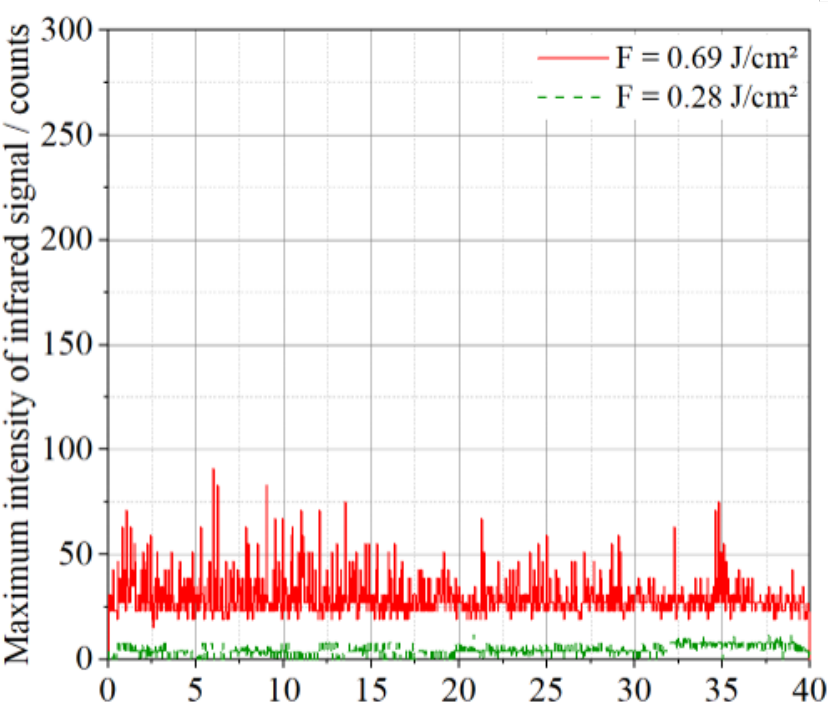

Fig. 4 Maximum infrared intensity of the signal as function of the process position during the laser treatment representing different fluences: a) for stainless steel (red solid line: $F=0.59 \mathrm{~J} / \mathrm{cm}^{2}$; green dashed line: $F=0.24 \mathrm{~J} / \mathrm{cm}^{2}$ ) with constant process parameters (Overlap $o_{P}=99.67 \%$, repetition rate $f=100 \mathrm{kHz}$ ) and b) for aluminum (red solid line: $F=0.69 \mathrm{~J} / \mathrm{cm}^{2}$; green dashed line: $F=0.28 \mathrm{~J} / \mathrm{cm}^{2}$ ) with constant process parameters (Overlap $o_{P}=99.67 \%$, repetition rate $f=100 \mathrm{kHz}$ ).

\section{Stainless steel}

Overlap $o_{P}=99.00 \%$ and Fluence $F=0.24 \mathrm{~J} / \mathrm{cm}^{2}$ :

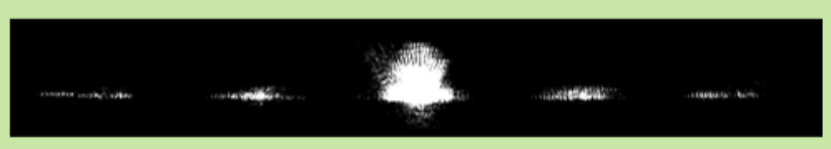

Overlap $o_{P}=99.00 \%$ and Fluence $F=0.36 \mathrm{~J} / \mathrm{cm}^{2}$ :

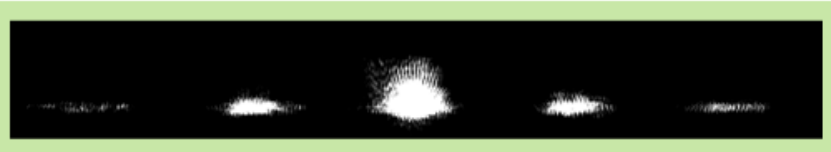

Overlap $o_{P}=99.67 \%$ and Fluence $F=0.47 \mathrm{~J} / \mathrm{cm}^{2}$ :

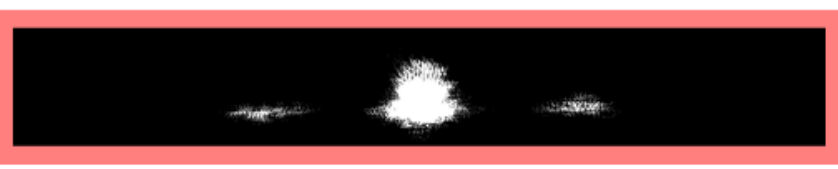

Overlap $o_{P}=99.67 \%$ and Fluence $F=0.59 \mathrm{~J} / \mathrm{cm}^{2}$ :

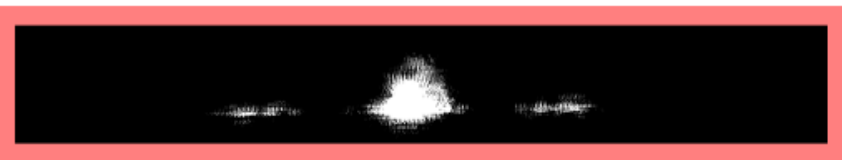

\section{$\underline{\text { Aluminum }}$}

Overlap $o_{P}=99.00 \%$ and Fluence $F=0.28 \mathrm{~J} / \mathrm{cm}^{2}$ :

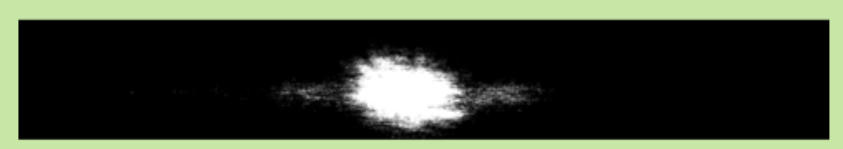

Overlap $o_{P}=99.00 \%$ and Fluence $F=0.41 \mathrm{~J} / \mathrm{cm}^{2}$ :

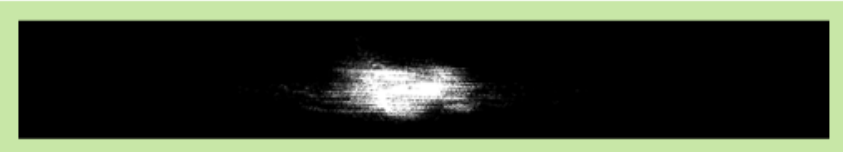

Overlap $o_{P}=99.67 \%$ and Fluence $F=0.55 \mathrm{~J} / \mathrm{cm}^{2}$ :

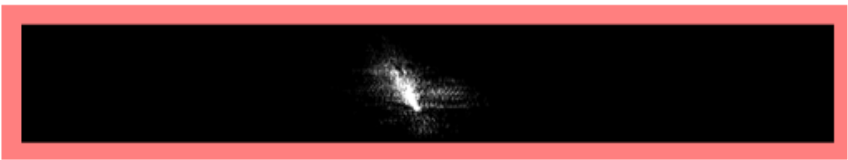

Overlap $o_{P}=99.67 \%$ and Fluence $F=0.69 \mathrm{~J} / \mathrm{cm}^{2}$

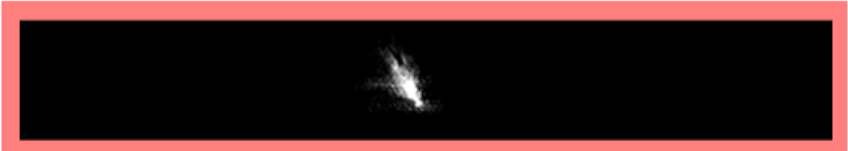

Fig. 5 Selected resulting images of the diffraction patterns for stainless steel (left) and aluminum samples (right), captured with the diffraction measurement system. The background color in the figure represents two obtained surface conditions: green - good surface quality; red - bad surface quality.

absorbed energy of the laser and the dissipated energy were balanced. In contrast, the infrared signal measured during the laser treatment at $0.24 \mathrm{~J} / \mathrm{cm}^{2}$ laser fluence, was approximately four times lower and remained constant along the processed track without significant fluctuations during the process.
Similarly as in the case of steel, the intensity captured by the MWIR camera during the laser treatment was evaluated, too. Figure $4 \mathrm{~b}$ shows the infrared signals for the laser processing of aluminum samples at 0.28 and $0.69 \mathrm{~J} / \mathrm{cm}^{2}$. Contrary to the experiments with stainless steel, the signal intensity remained on a constant level during the laser treatment 
Diffraction order:

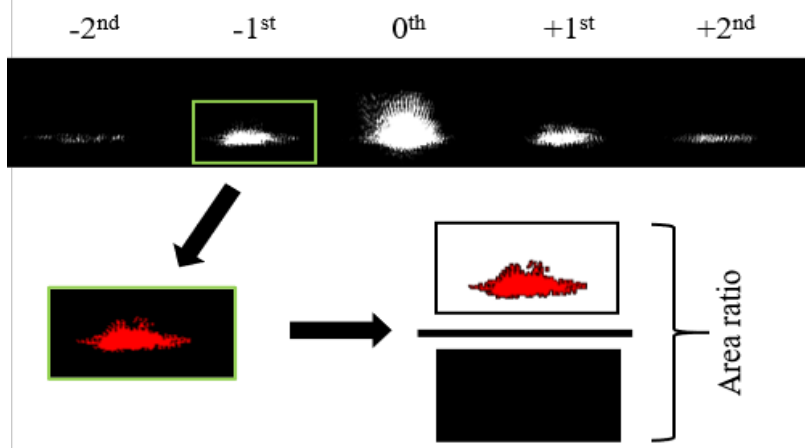

Fig. 6 Schematic process of the analysis algorithm to evaluate the diffraction image with its different diffraction orders.

also at high fluence $\left(0.69 \mathrm{~J} / \mathrm{cm}^{2}\right)$. Furthermore, the captured intensity was significantly lower (at least four times) compared to stainless steel. The higher thermal conductivity of aluminum might explain this behavior $(15 \mathrm{~W} / \mathrm{m} \cdot \mathrm{K}$ and $119 \mathrm{~W} / \mathrm{m} \cdot \mathrm{K}$ for steel and aluminum, respectively) [36,37].
This suggests that the heat, which is introduced in the material with the lowest thermal conductivity, cannot be dissipated efficiently and quickly from the process zone to the surrounding material. Furthermore, the emissivity of aluminum is significantly lower compared to stainless steel (e.g. 0.06 and 0.19 for aluminum and stainless steel, respectively) and thus a lower emission intensity can be expected for the former material [38]. Similarly as for stainless steel, for the lower laser fluence, a relatively lower intensity and the infrared signal was detected and the intensity level was constant during the laser treatment. The structures produced at these energy levels $\left(0.24\right.$ and $\left.0.28 \mathrm{~J} / \mathrm{cm}^{2}\right)$ presented a high quality and uniformity of the line-like pattern geometry.

Subsequent to the structuring process, the treated samples were evaluated with the diffraction measurement system (scatterometry system). Figure 5 shows different examples of the recorded signals depending on pulse overlap, laser fluence and material. Clearly, different patterns were obtained as a result of the dissimilar surface conditions. In particular for the treated steel samples, the images showed the different diffraction orders, whose position on the camera can be correlated with the number of the diffraction order as well as with the spatial period of the produced pattern. The

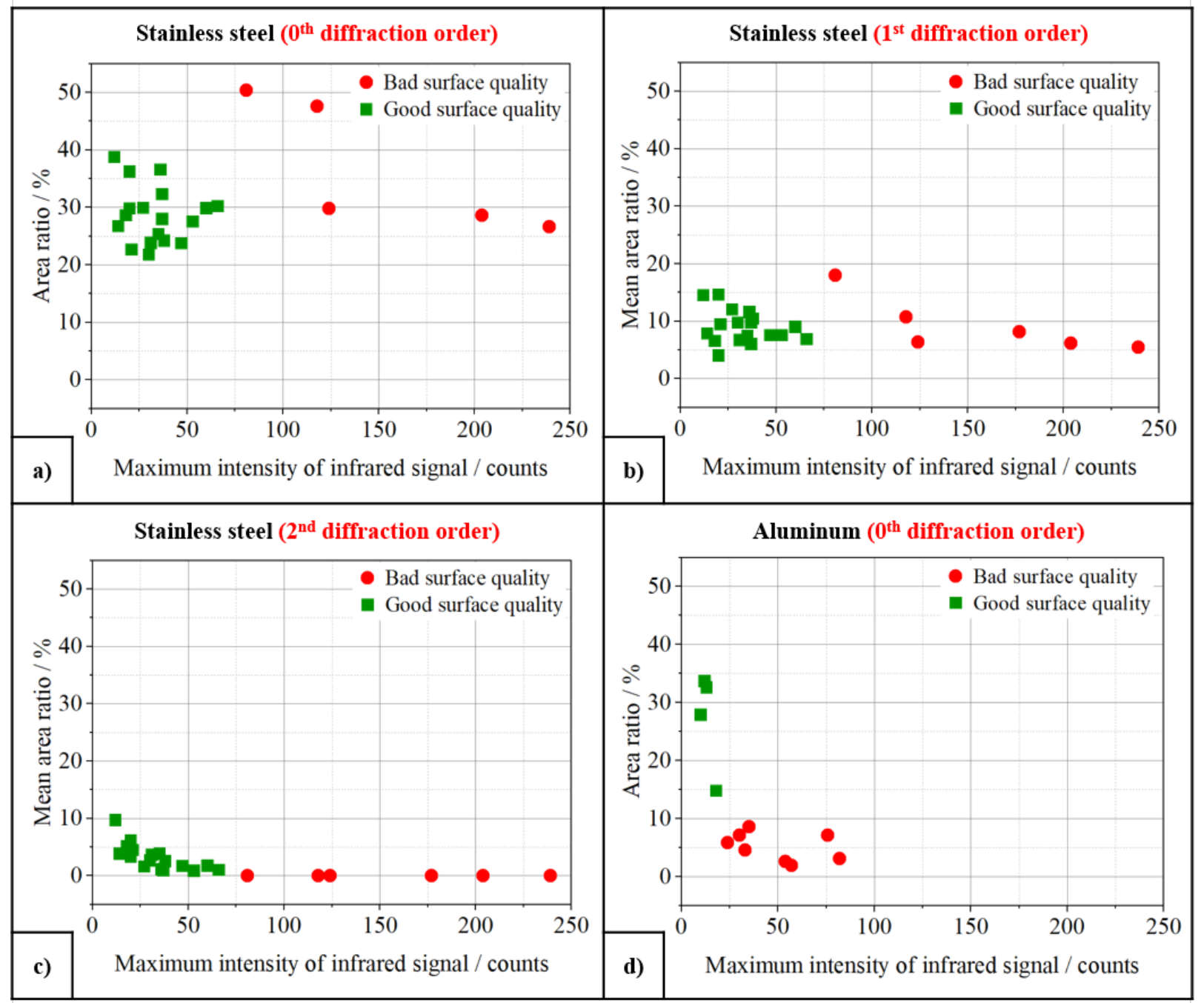

Fig. 7 Mean area ratio as a function of the maximum intensity of infrared signal for $0^{\text {th }}, \pm 1^{\text {st }}$ and $\pm 2^{\text {nd }}$ diffraction order. In the charts, the surfaces in a good (green squares) and bad condition (red circles) are shown. Charts (a-c) correspond to stainless steel samples and (d) to aluminum. The mean area ratio was calculated with the area ratio of the corresponding positive and negative diffraction order in the image. 
fields highlighted with green background indicate good surface conditions (isotropy I lower than $2.3 \%$ ) without excessive molten material as has been discussed before. Contrarily, the surface quality of fields with red background color are characterized by surface topographies with an excessive amount of molten material over the produced line-like structure on the metallic samples. As discussed in previous study [30], the area of the captured diffraction orders can be used for analyzing the different geometries of a line-like structure. In the case of the stainless steel samples, the $0^{\text {th }}, \pm 1^{\text {st }}$ and $\pm 2^{\text {nd }}$ diffraction orders are visible, while for aluminum, only the zero order was observed (Figure 5). In addition, the area of the diffraction orders differs depending on the used process parameters (due to the different surface quality of the line-like patterns).

A quantitative analysis to evaluate the different diffraction images was carried out, following the strategy presented in Figure 6. The procedure started from the diffraction images caught by the diffraction measurement system. A rectangular frame was overlaid symmetrically on each diffraction order $m$. Afterwards, a gray scale analysis within the region of interest was performed. Thus, the ratio between the number of bright pixels (pixels with a grey scale value higher than zero) and the number of black pixels (with a grey scale value of zero) could be calculated. This analysis was accomplished for all diffraction orders $\left(m=-2^{\text {nd }}, m=-1^{\text {st }}\right.$, $\left.m=0^{\text {th }}, m=+1^{\text {st }}, m=+2^{\text {nd }}\right)$ of the images.

With the resulting data of both the infrared camera and the scatterometry system, it was evaluated which combination of parameters can be used to identify structures that have a good surface quality (isotropy value lower than $2.3 \%$ ). Figure 7 illustrates the ratio area of the $0^{\text {th }}, \pm 1^{\text {st }}$ and $\pm 2^{\text {nd }}$ diffraction order as a function of the intensity of the infrared signal captured by the MWIR camera for stainless steel and aluminum samples. The represented area of a diffraction order in the plots was calculated as an average value of the corresponding positive and negative diffraction order in the images. The condition of the resulting surface structure is highlighted with different shapes and colors. The red circles indicate a bad surface quality with significant amount of resolidified material as well as a deteriorated pattern structure, whereas the green squares are characterized for having a good surface condition with a homogenous pattern on the surface.

From the plots it is possible to deduce that there is a threshold value related with the intensity of the detected infrared signal, that distinguish deteriorated surface structures from homogeneous (well-aligned) ones for both materials. For stainless steel, an intensity level lower 66 counts describes the samples that have a good surface quality. For aluminum, the threshold intensity value was 18 counts.

A similar analysis was performed for the calculated area of the captured diffraction orders. In case of the stainless steel samples, the area ratio of the $0^{\text {th }}$ (Figure $7 \mathrm{a}$ ) and $\pm 1^{\text {st }}$ diffraction orders (Figure $7 b$ ) do not provide a satisfactory criterion for drawing conclusions on the quality of the surface pattern. For example, area ratios between 20 and $40 \%$ were observed for surface structures with arbitrary good or bad qualities. For the $\pm 1^{\text {st }}$ diffraction order the same behavior was observed, with area ratios between 4 and $15 \%$. Differently, the analysis of the $\pm 2^{\text {nd }}$ diffraction order (Figure 7c) provides reliable criteria to clearly distinguish the two surface conditions. In this case, patterned surfaces providing area ratios of $0 \%$ were only related to structures with a bad surface quality (such as in Figure 5, for $o_{P}=99.67 \%$ and $F=0.59 \mathrm{~J} / \mathrm{cm}^{2}$ ). This implies that, for structures with a bad surface quality, the $\pm 2^{\text {nd }}$ diffraction order were not visible. Consequently, if the area ratio of the $\pm 2^{\text {nd }}$ diffraction order is higher than zero, these structured surfaces denote a good quality.

In contrast to stainless steel, for all treated aluminum samples, only the zero diffraction order was visible. The reason for this behavior is not fully understood yet. A possible explanation could be related to the formation of oxides during the laser treatment, as well as the significant darkening of the aluminum surfaces due to the laser treatment. Therefore, in future investigations a laser source with higher power will be used in the diffraction measurement system developed (Figure 2) in order to increase the intensity of the reflected light and thus allowing at least the $\pm 1^{\text {st }}$ diffraction order to be visualized.

In consequence, only the zero diffraction order was analyzed (Figure 7d). Differently from the stainless steel substrates, in this case it was observed an increase of the intensity of infrared signal for patterns showing a good quality. The threshold of the area ratio for this case was $14 \%$, which indicates that above this threshold, surfaces with sufficient pattern quality can be identified.

Considering both monitoring approaches presented in this work, it can be inferred that the thresholds of both the intensity of the infrared signal and the relative area of the diffractions orders can be utilized to clearly determine periodic surface structures with significant quality. For the stainless steel surfaces, the recorded infrared signal and the area (or relative intensity) of the $\pm 2^{\text {nd }}$ diffraction orders are sufficient to distinguish between good and bad qualities. In case of aluminum, the zero diffraction order has to be considered, in combination with the infrared signal. Nevertheless, the captured information does not allow to determine topographical further parameters like relative roughness or structure depth. Therefore, additional investigations are necessary in the future to elucidate these questions.

\section{Conclusions}

In this work, two different innovative monitoring systems have been proposed in order to evaluate the quality of periodic structures produced by DLIP. The used systems consisted on a MWIR camera recording the infrared signal during the laser treatments as well as a self-developed diffraction measurement system capable to examine precisely periodic surface patterns.

Firstly, the isotropy factor was used to evaluate the quality of the produced patterns quantitatively. For both used materials, values lower than $2.3 \%$ corresponded to periodic structures not showing resolidified material induced by excessive energy provided by the laser during the DLIP treatment.

The MWIR camera could accurately observe the resulting infrared radiation during the DLIP process for both stainless steel and aluminum samples. The infrared intensity showed a strong dependence on the quality of the obtained surfaces. In addition, the diffraction measurement system 
was able to capture the images with the characteristic signature (e.g. diffraction pattern) of the produced structure. The combination of both methods allowed to determine the following criteria for structure quality evaluation depending on the used material:

(i) for stainless steel, structures with an adequate quality were obtained when the intensity of the infrared signal was lower than 66 counts and the area ratio for the $\pm 2^{\text {nd }}$ diffraction orders was higher than $0 \%$.

(ii) for aluminum, structures with an adequate quality were obtained when the intensity of the infrared signal was lower than 18 counts and the area ratio for the zero diffraction order was higher than $14 \%$.

In conclusion, the parameters described above allow the definition of an effective criteria for distinguishing high quality DLIP-structures. In the future, monitoring of aluminum substrates could be improved by utilizing a laser source with a higher power, allowing to record at least the $\pm 1^{\text {st }}$ diffraction orders. Further aspects should also be implemented in order to determine quantitative information of the produced topographies during the structuring process.

\section{Acknowledgments}

This project has received funding from the European Union's Horizon 2020 research and innovation programme under Grant Agreement No. 825132. It is an initiative of the Photonics Public Private Partnership www.photonics21.org. This work reflects only the author's view and the EU is not responsible for any use that may be made of the presented information.

\section{References}

[1] T. B. H. Schroeder, J. Houghtaling, B. D. Wilts, and M. Mayer: Adv. Mater., 30, (2018) 1705322.

[2] C. Zhang, D. A. Mcadams, and J. C. Grunlan: Adv. Mater., 28, (2016) 6292.

[3] G. Liu, Z. Yuan, Z. Qiu, S. Feng, Y. Xie, D. Leng, and X. Tian: Ocean Eng., 199, (2020) 106962.

[4] E. Liu, H. J. Lee, and X. Lu: Appl. Sci., 10, (2020) 2678.

[5] R. S. S. Raja, P. Selvakumar, and P. D. Babu: J. Mech. Sci. Technol, 34, (2020) 1667.

[6] Z. Yang, C. Zhu, N. Zheng, D. Le, and J. Zhou: Materials, 11, (2018) 2210.

[7] A. H. A. Lutey, L. Gemini, L. Romoli, G. Lazzini, F Fuso, M. Faucon, and R. Kling: Sci Rep, 8, (2018) 10112

[8] F. H. Rajab, C. M. Liauw, P. S. Benson, L. Li, and K. A. Whitehead: Colloids Surf. B, 160, (2017) 688.

[9] F. H. Rajab, C. M. Liauw, P. S. Benson, L. Li, and K. A. Whitehead: Food Bioprod. Process., 109, (2018) 29.

[10] S. Maragkaki, C. A. Skaradzinski, R. Nett, and E. L. Gurevich: Sci Rep, 10, (2020) 53.

[11] B. Voisiat, W. Wang, M. Holzhey, and A. F. Lasagni: Sci Rep, 9, (2019) 7801.

[12] D. W. Müller, T. Fox, P. G. Grützmacher, S. Suarez, and F. Mücklich: Sci Rep, 10, (2020) 3647.

[13] A. Lasagni, D. Benke, T. Kunze, M. Bieda, S. Eckhardt, T. Roch, D. Langheinrich, and J. Berger: J. Laser Micro Nanoeng., 10, (2015) 340.
[14] Y. Nakata: Adv. Opt. Technol., 5, (2016) 29.

[15] S. Indrišiūnas, B. Voisiat, M. Gedvilas, and G. Račiukaitis: J. Laser Appl., 29, (2017) 011501.

[16] A. F. Lasagni, P. Shao, J. L. Hendricks, C. M. Shaw, D. C. Martin, and S. Das: Appl. Surf. Sci., 256, (2010) 1708.

[17] M. Weck and C. Brecher: in Werkzeugmaschinen 3, Springer Berlin Heidelberg, Berlin, Heidelberg, (2006), pp. 267-404.

[18] T. Purtonen, A. Kalliosaari, and A. Salminen: Phys. Procedia, 56, (2014) 1218.

[19] L. Zheng, Q. Zhang, H. Cao, W. Wu, H. Ma, X. Ding, J. Yang, X. Duan, and S. Fan: Mater. Des. 183, (2019) 108110.

[20] M. Y. Kayacan and N. Y1lmaz: Measurement, 160, (2020) 107825.

[21] A. Ancona, V. Spagnolo, P. M. Lugarà, and M. Ferrara: Appl. Opt., 40, (2001) 6019.

[22] M. Sokolov, P. Franciosa, T. Sun, D. Ceglarek, V. Dimatteo, A. Ascari, A. Fortunato, and F. Nagel: J. Laser Appl., 33, (2021) 012028.

[23] F. Bauer, A. Michalowski, T. Kiedrowski, and S. Nolte: Opt. Express, 23, (2015) 1035.

[24] J. H. Pak, S. Jeong, T. Lim, and S. Ju: Adv. Opt. Mater., 8, (2020) 1901706.

[25] D. V. Tran, Y. C. Lam, H. Y. Zheng, B. S. Wong, and D. E. Hardt: Appl. Surf. Sci., 253, (2007) 7290.

[26] D. V. Tran, Y. C. Lam, B. S. Wong, H. Y. Zheng, and D. E. Hardt: Opt. Express, 14, (2006) 9261.

[27] K. Chen and T. Sun: Asia-Pac. J. Chem. Eng., 12, (2017) 307.

[28] L. R. de Lara, R. Jagdheesh, and J. L. Ocaña: Mater. Lett., 184, (2016) 100.

[29] M. H. Madsen and P.-E. Hansen: Opt. Express, 24, (2016) 1109.

[30] S. Teutoburg-Weiss, B. Voisiat, M. Soldera, and A. F. Lasagni: Materials, 13, (2019) 53.

[31] C. Alleaume, S. Alamri, T. Kunze, J. Ziegler, A. Wilson, and R. Bola: J. Phys. Photonics, 2, (2020) 031004.

[32] J. Turunen and T. Vallius: in Encyclopedia of Modern Optics (Ed.: R.D. Guenther), Elsevier, Oxford, (2005), pp. 229-239.

[33] R. Rosenholtz and J. Malik: Vision Res., 37, (1997) 2283.

[34] K. Stout: The Development of Methods for the Characterisation of Roughness in Three Dimensions, (ECSC, EEC, EAEC, Brussels-Luxembourg, 1993).

[35] N. Schröder, G. Vergara, B. Voisiat, and A. F. Lasagni: J. Laser Micro Nanoeng., 15, (2020) 150.

[36] S. Ertl: Investigation on the production and use of microtechnically manufactured diamond tools, Dissertation, University of Freiburg, (2003).

[37] R. C. Batra and C. H. Kim: Int. J. Plast., 8, (1992) 425.

[38] Transmetra GmbH: Table of Emissivity of Various Surfaces For Infrared Thermometry, https://www.transmetra.ch/images/transmetra_pdf/publikationen_literatur/pyrometrie-thermografie/emissivity_table.pdf.

(Received: June 10, 2021, Accepted: October 19, 2021) 